\title{
Effective Spin Foam Models for Four-Dimensional Quantum Gravity
}

\author{
Seth K. Asante, ${ }^{1,2}$ Bianca Dittrich ${ }^{1}{ }^{\text {and Hal M. Haggard }}{ }^{3,1}$ \\ ${ }^{1}$ Perimeter Institute, 31 Caroline Street North, Waterloo, ON, N2L 2Y5, CAN \\ ${ }^{2}$ Department of Physics and Astronomy, University of Waterloo, \\ 200 University Avenue West, Waterloo, Ontario, Canada, N2L 3G1 \\ ${ }^{3}$ Physics Program, Bard College, 30 Campus Road, Annandale-On-Hudson, NY 12504, USA
}

\begin{abstract}
A number of approaches to four-dimensional quantum gravity, such as loop quantum gravity and holography, situate areas as their fundamental variables. However, this choice of kinematics can easily lead to gravitational dynamics peaked on flat spacetimes. We show that this is due to how regions are glued in the gravitational path integral via a discrete spin foam model. We introduce a family of 'effective' spin foam models that incorporate a quantum area spectrum, impose gluing constraints as strongly as possible, and leverage the discrete general relativity action to specify amplitudes. These effective spin foam models avoid flatness in a restricted regime of the parameter space.
\end{abstract}

Symplectic, metrical quantization. Envisioning the geometry of spacetime as dynamically evolving founded the revolutionary insights of general relativity (GR) that have resulted in direct measurements of gravitational time dilation, bending of starlight, and gravitational waves. However, this revolution remains incomplete. We still do not know how to fully characterize an evolving quantum spacetime geometry.

The quantization of spacetime geometry is an interplay between its symplectic and metrical aspects. The former determines the allowed phase space and its associated quantum theory, while the latter encodes spacetime dynamics. In three dimensions (3D) alignment between these two facets of geometry allows one to construct a discrete, simplicial path integral for quantum gravity, the Ponzano-Regge model [1]. Spacetime is decomposed into a large collection of tetrahedra that are glued along a subset of edges with matched lengths. Metrical and symplectic aspects of this geometry nicely align: lengths encode the intrinsic metric and tedrahedral dihedral angles encode the extrinsic geometry and these variables are canonically conjugated to each other $[2,3]$. In the Euclidean signature case the angles are compact, which leads to discrete spectra for the lengths.

In $4 \mathrm{D}$ the situation is more subtle, and there is some tension between the symplectic and the metrical. In a space-time split, the metric has two natural discretizations: the lengths of edges, and the extrinsic curvature angles defined around 2D faces.

These variables are not canonically conjugate. This forces a choice: either the lengths or the extrinsic curvature angles must be completed to a set of canonically conjugated coordinates.

If the lengths are chosen, then the conjugate variables are contractions of the curvature angles with certain arealength derivatives [4]. These variables have, so far, resisted rigorous quantization. Meanwhile for the curvature angles, the conjugate variables are the $2 \mathrm{D}$ face areas, whose quantization give a discrete area spectrum; this is because they are conjugate to compact angles. These variables arise naturally in connection formulations of GR, like Loop Quantum Gravity (LQG) [5]. A key result of LQG is the rigorous quantization of area and volume observables, which indeed have discrete spectra [6-9].

In spin foam models [10-12], which are discrete geometry path integrals derived from LQG, area variables are fundamental. Area variables play a central role in holography $[13,14]$, in particular, for the reconstruction of geometry from entanglement $[15,16]$, and discrete area spectra are key in many approaches to black hole entropy counting [17-20]. There is, however, an interplay between the choice of area variables and the dynamics of GR: in this letter we show that area variables must be constrained to avoid a suppression of curvature and that the discreteness of their spectra hinders sharp imposition of these constraints.

In fact, it has been argued that, in the semiclassical limit, flat configurations dominate the spin foam path integral [21-27]. We reveal the mechanism behind this unfortunate dominance, and identify a more favorable regime in which the path integral can peak on curved configurations. This 'flatness problem' has been a key open problem for spin foams [27]. We show that it can be traced back to fundamental, discrete, area variables.

We tackle directly the question of whether a discrete, locally independent, area spectrum is consistent with the dynamics of GR. To this end we propose a family of 'effective' models that (a) incorporate a discrete area spectrum, (b) impose the constraints between the areas as strongly as allowed by the LQG Hilbert space structure, and (c) use - more directly than current spin foam models - a discretized GR action for the amplitudes.

These effective models allow us to show that the flatness problem can be overcome, but to do so also imposes certain restrictions involving the discretization scale, curvature per triangle, and the Barbero-Immirzi parameter, which controls the area spectral spacing. Future work will show whether these restrictions are sufficient to ensure general relativistic dynamics in the continuum limit. 
Discrete, locally-independent areas. We study a path integral for $4 \mathrm{D}$ quantum gravity regulated by a triangulation of spacetime. We work with quantum amplitudes for Euclidean signature, leaving the Lorentzian case to future work. Our key assumption is that the areas have a discrete, prescribed spectrum. We take the area eigenvalues to be independent, that is, their values will not depend on the state away from the measured triangle. The particular area spectrum we work with is

$$
a(j)=\gamma \ell_{P}^{2} \sqrt{j(j+1)} \sim \gamma \ell_{P}^{2}(j+1 / 2),
$$

where $j$ is a half-integer spin label, $\ell_{P}=\sqrt{8 \pi \hbar G / c^{3}}$ is the Planck length, $\gamma$ is the dimensionless BarberoImmirzi parameter, and $\sim$ indicates the large- $j$ asymptotic limit. We focus on the equispaced asymptotic spectrum. This form for the area spectrum was established in LQG [6-9], but discrete areas have also been discussed in the context of black hole spectroscopy [17]. In LQG, triangle normals are represented by angular momentum vector operators rescaled by $\gamma[28,29]$. The area is thus given by the square root of the $\mathrm{SU}(2)$ Casimir, multiplied by $\gamma$, which determines the area eigenvalue spacing.

Before taking up the path integral, we review the use of area variables in simplicial discretizations of GR. These discretizations were introduced by Regge [30] and used length variables. A wide array of reformulations have been considered [31-36], and we use descriptive adjectives to capture the variables used in each form. The change from length to area variables turns out to be more subtle than one might expect. A treatment in the more transparent context of Regge calculus will illuminate the issues before discussing the path integral.

Actions for discretized GR. In Length Regge Calculus (LRC) one replaces the metric by lengths $l_{e}$ assigned to the edges $e$ of a triangulation. The $l_{e}$ determine the triangle areas $A_{t}(l)$ and the $4 \mathrm{D}$ (internal) dihedral angles $\theta_{t}^{\sigma}(l)$ in each 4 -simplex $\sigma$. The action

$$
S_{\mathrm{LRC}}=\sum_{t} n_{t} \pi A_{t}-\sum_{\sigma} \sum_{t \supset \sigma} A_{t} \theta_{t}^{\sigma} \equiv \sum_{t} S_{t}^{l}+\sum_{\sigma} S_{\sigma}^{l}
$$

is a discretization of the Einstein-Hilbert action and the corresponding equations of motion approximate Einstein's equations [37]. The factor $n_{t} \in\{1,2\}$ allows for triangulations with boundary and is 1 for triangles on the boundary and 2 for triangles in the bulk.

The 4-simplices, which are the basic building blocks of the triangulation, each have 10 edges and 10 areas. One can thus (locally) invert the 10 functions $A_{t}(l)$ that give a simplex's areas in terms of its lengths [38]. We will denote the resulting functions $L_{e}^{\sigma}(a)$, where $a$ collectively signifies the 10 areas associated to $\sigma$. This allows us to define the Area Regge Calculus (ARC) action [31, 32, 36], whose value on configurations with $a_{t}=A_{t}(l)$ agrees with the LRC action

$$
S_{\mathrm{ARC}}=\sum_{t} S_{t}^{a}(a)+\sum_{\sigma} S_{\sigma}^{a}(a)
$$

where $S_{t}^{a}(a)=n_{t} \pi a_{t}$ and $S_{\sigma}^{a}(a)=S_{\sigma}^{l}\left(L^{\sigma}(a)\right)$. Strikingly, freely varying the bulk areas one finds that the deficit angles $\epsilon_{t}=2 \pi-\sum_{\sigma \supset t} \theta_{t}^{\sigma}$, which measure curvature, have to vanish [36]. That is, the ARC equations of motion impose flatness.

Extended triangulations are built up by gluing pairs of 4-simplices $\left(\sigma, \sigma^{\prime}\right)$ through a shared tetrahedron $\tau$. Gluing identifies six pairs of length variables but only four pairs of area variables, which explains why the equations of motion for LRC and ARC differ: after gluing there are generically more triangles than edges in a $4 \mathrm{D}$ triangulation and thus more area than length variables. Restricting variation of the areas to a constraint surface coming from a consistent length assignment $a_{t}=A_{t}(l)$, one recovers the LRC equations of motion.

From this counting we see that working with area variables we miss two matching conditions per bulk tetrahedron. The geometry of a tetrahedron, however, can be uniquely specified by its four areas and two 3D dihedral angles at non-opposite edges (intriguingly, opposite dihedral angles do not suffice). Introducing the 3D dihedral angles $\Phi_{e}^{\tau, \sigma}(a)=\Phi_{e}^{\tau}\left(L^{\sigma}(a)\right)$, we have thus, two constraints per bulk tetrahedron

$$
\Phi_{e_{i}}^{\tau, \sigma}(a)-\Phi_{e_{i}}^{\tau, \sigma^{\prime}}(a) \stackrel{!}{=} 0 \quad i=1,2,
$$

where $\left(e_{1}, e_{2}\right)$ is a choice of a pair of non-opposite edges in $\tau$. Together with the matched areas, these constraints ensure that the lengths of a shared tetrahedron, as defined by the areas associated to $\sigma$ and $\sigma^{\prime}$, match.

The constraints (4) involve pairs of neighbouring simplices. Introducing two $3 \mathrm{D}$ dihedral angles $\phi_{e_{i}}^{\tau}$ per tetrahedron as additional variables [35], we can formulate alternative constraints, localized on a given 4 -simplex $\sigma$ :

$$
\phi_{e_{i}}^{\tau}-\Phi_{e_{i}}^{\tau, \sigma}(a) \stackrel{!}{=} 0 \quad i=1,2 .
$$

These constraints fix all variables $\phi_{e_{i}}^{\tau}$ as functions of the areas and impose the constraints (4). We favor these localized constraints because they preserve the additive factorization of the action (3) and lead to the simplifying product factorization of the path integral below, Eq. (8).

In the quantum theory, areas are encoded using $\mathrm{SU}(2)$ representation labels $j_{t}$, cf. Eq. (1), which result from identifying triangle normals with angular momentum operators. The 3D dihedral angles are given by the inner product of these normals, and can be encoded in the recoupling of two angular momenta. The angles at a pair of non-opposite edges $\left(e_{1}, e_{2}\right)$ in a tetrahedron $\tau$ require different recoupling schemes and are therefore noncommutative [39, 40] (for a simplified proof see [41]):

$$
\hbar\left\{\phi_{e_{1}}^{\tau}, \phi_{e_{2}}^{\tau}\right\}=\ell_{P}^{2} \gamma \frac{\sin \alpha_{v}^{t, \tau}}{a_{t}}=\frac{\sin \alpha_{v}^{t, \tau}}{\left(j_{t}+\frac{1}{2}\right)},
$$

where $\alpha_{v}^{t, \tau}$ is the angle between $\left(e_{1}, e_{2}\right)$. Thus, the constraints (5) are also non-commutative, more precisely, 
second class. For these second class constraints the uncertainty relations prevent a sharp imposition of the constraints in the quantum theory. Armed with these understandings, we take up the path integral.

Path integral. To incorporate a discrete area spectrum (1), we employ constrained ARC, and sum over spin labels $j_{t}$ :

$$
\mathcal{Z}=\sum_{\left\{j_{t}\right\}} \mu(j) \prod_{t} \mathcal{A}_{t}(j) \prod_{\sigma} \mathcal{A}_{\sigma}(j) \prod_{\tau \in \mathrm{blk}} G_{\tau}^{\sigma, \sigma^{\prime}}(j) .
$$

The triangle $\mathcal{A}_{t}=\exp \left(\mathrm{i} \gamma n_{t} \pi\left(j_{t}+\frac{1}{2}\right)\right)$ and simplex amplitudes $\mathcal{A}_{\sigma}=\exp \left(-\mathrm{i} \gamma \sum_{t \in \sigma}\left(j_{t}+\frac{1}{2}\right) \theta_{t}^{\sigma}(j)\right)$ result from the exponentiated ARC action (3). The precise form of the measure factor $\mu(j)$ will not be important for the discussion here, but see [46]. The factors $G_{\tau}^{\sigma, \sigma^{\prime}}$ implement the constraints (4), and are crucial for imposing the dynamics of LRC instead of the flat dynamics of ARC.

However, imposing the constraints (4) sharply, i.e., setting $G_{\tau}^{\sigma, \sigma^{\prime}}(j)=1$ if the constraints are satisfied, and $G_{\tau}^{\sigma, \sigma^{\prime}}(j)=0$ otherwise, leads to a severe problem: as we allow only discrete values for the areas, the constraints (4) constitute diophantine conditions. These conditions can only be satisfied for a very small set of labels with accidental symmetries, e.g., if all 10 pairs of labels match [41]. The resulting reduction in the density of states prevents a reasonable quantum dynamics. This obstacle has also been encountered in higher gauge formulations of gravity [47-50].

One way out is to weaken the constraints (4), e.g., by allowing a certain error interval. But, one has to navigate between Scylla - reducing too much the density of states - and Charybdis - imposing a dynamics that does not match GR.

Here we will take guidance from LQG and impose the constraints as strongly as allowed by the uncertainty relations resulting from (6). To this end we employ states that are coherent in the two angle variables per tetrahedron, but restrict to the eigenspaces for the area operators. There are different constructions available for such states [43, 51-53]. For a given tetrahedron $\tau$ we will denote the coherent states $\mathcal{K}_{\tau}\left(\phi_{e_{i}}^{\tau} ; \Phi_{e_{i}}^{\tau, \sigma}\right)$, where $\phi_{e_{i}}^{\tau}=\left(\phi_{e_{1}}^{\tau}, \phi_{e_{2}}^{\tau}\right)$ are the arguments of the wave functions and $\Phi_{e_{i}}^{\tau, \sigma}=\left(\Phi_{e_{1}}^{\tau, \sigma}, \Phi_{e_{2}}^{\tau, \sigma}\right)$ are the angles on which the wave function is peaked. The coherent states come with a measure $d \mu_{\mathcal{K}}^{\tau}\left(\phi_{1}, \phi_{2}\right)$, the precise form of which is immaterial here. These coherent states can be used to define the path integral

$$
\mathcal{Z}^{\prime}=\sum_{\left\{j_{t}\right\}} \mu(j) \int \prod_{\tau} d \mu_{\mathcal{K}}^{\tau}(\phi) \prod_{t} \mathcal{A}_{t}(j) \prod_{\sigma} \mathcal{A}_{\sigma}^{\prime}(j, \phi),
$$

where the new simplex amplitude is given by

$$
\mathcal{A}_{\sigma}^{\prime}(j, \phi)=\mathcal{A}_{\sigma}(j) \prod_{\tau \in \sigma} \mathcal{K}_{\tau}\left(\phi_{e_{i}}^{\tau} ; \Phi_{e_{i}}^{\tau, \sigma}(j)\right) .
$$

Integrating out the angles we regain-without approximation and modulo boundary contributions [54] — a path integral of the form (7) where the factors $G_{\tau}^{\sigma, \sigma^{\prime}}$ are given by inner products between coherent states peaked on the angles in $\tau$ induced by the areas of $\sigma$ and $\sigma^{\prime}$, respectively,

$$
G_{\tau}^{\sigma, \sigma^{\prime}}(j)=\left\langle\mathcal{K}_{\tau}\left(\cdot ; \Phi_{e_{i}}^{\tau, \sigma}(j)\right) \mid \mathcal{K}_{\tau}\left(\cdot ; \Phi_{e_{i}}^{\tau, \sigma^{\prime}}(j)\right)\right\rangle .
$$

By construction, this inner product is peaked on the matching conditions (4) and provides a precise sense in which they are weakly imposed. Imposition of these constraints leads to the brackets (6), and through them to our main results (12) and (13). Counting the spin configurations contained in different confidence intervals of the $G_{\tau}^{\sigma, \sigma^{\prime}}$ factors suggests that the weak imposition of the area constraints leads to a reasonable number of configurations contributing to the path integral (7), see [41].

On the flatness problem. We consider a first test case for the dynamics encoded in the path integral (7). We choose a triangulation where we can control the scale for the bulk area variable and the bulk curvature through the boundary data. The complex consists of three 4simplices sharing a single bulk triangle. There are no bulk edges, thus no bulk variables to sum over in LRC, and the bulk deficit angle is determined by the boundary lengths. Nonetheless in ARC, there is one bulk variable to sum over, which imposes a vanishing deficit angle for the internal triangle in the unconstrained theory.

The amplitude of the path integral consists of two pieces: (i) an oscillatory phase factor, given by the exponentiated ARC action and (ii) the $G$-factors, which are peaked on the area constraints (4) and decay exponentially. We employ scaling arguments and approximate these factors by gaussians with deviation,

$$
\sigma(\Phi) \sim \sqrt{\sin \alpha_{v}^{t, \tau} /\left(j_{t}+\frac{1}{2}\right)},
$$

determined by the Poisson brackets (6).

The semiclassical limit, as usually understood for spin foams, amounts to a large $j$ limit. The reason for this is the linear scaling of the action (3) in the areas, and thus in the spins $j$. As the amplitudes include an oscillatory and an exponentially decaying factor, there are two conditions for configurations to contribute [21, 55]: (i) the oscillatory phase should be stationary and (ii) the $G$-factors should be near their maxima. As the oscillatory phase is given by the exponentiated Area Regge action, the stationarity condition (i) leads to flatness in the unconstrained model. A similar structure of the amplitudes, and hence a similar flatness problem [21-27], arises for the EPRL-FK spin foam models [12].

In the following we will perform a more detailed analysis and identify a regime in which curved configurations can dominate. A bound, that has heretofore received little attention, emerges. We distinguish the spin scales set by the boundary, $j$, and by the bulk, $j_{\text {blk }}$. For the minimal triangulations investigated here $j \sim j_{\text {blk }}$. 
Since we are interested in the semiclassical limit, we focus on the classical action and on the exponent of the gaussian $G$-factors. The small $\hbar$ limit of these exponents are dominated by the classical values, from which our scaling results will follow. From (11) we see that the $G$ factors come with a deviation $\sigma(\Phi) \sim 1 / \sqrt{j}$ for the 3D dihedral angle, where we assume that the boundary areas $a \sim \gamma \ell_{P}^{2} j$ have approximately equal values, so that the bulk area scales as $a_{\mathrm{blk}} \sim a$. As angles are dimensionless, their derivatives scale as $\partial \Phi / \partial a_{\mathrm{blk}} \sim 1 / a$ and $\partial \epsilon / \partial a_{\mathrm{blk}} \sim$ $1 / a$. Thus, we have $\partial \Phi / \partial j_{\text {blk }} \sim 1 / j$ and $\partial \epsilon / \partial j_{\text {blk }} \sim 1 / j$, and the deviations of the gaussian $G$-factors, expressed as functions of bulk spin and deficit angle $\epsilon$, respectively, scale as

$$
\begin{aligned}
& \sigma\left(j_{\mathrm{blk}}\right) \sim\left[\frac{\partial \Phi\left(j_{\mathrm{blk}}\right)}{\partial j_{\mathrm{blk}}}\right]^{-1} \times \sigma(\Phi) \sim j \times \frac{1}{\sqrt{j}}=\sqrt{j}, \\
& \sigma(\epsilon) \sim\left[\frac{\partial \epsilon\left(j_{\mathrm{blk}}\right)}{\partial j_{\mathrm{blk}}}\right] \times \sigma\left(j_{\mathrm{blk}}\right) \sim \frac{1}{j} \times \sqrt{j}=\frac{1}{\sqrt{j}} .
\end{aligned}
$$

As the angles are invariant under rescaling, we can choose boundary data that induce a given deficit angle $\epsilon$, and then choose a sufficiently large scale $j$, so that the $\epsilon=0$ value is outside the deviation interval. Thus by going to sufficiently large spins $j$, the constraint part of the amplitudes can peak sharply on non-vanishing curvatures. Note that the deviations $\sigma\left(j_{\mathrm{blk}}\right)$ and $\sigma(\epsilon)$ as functions of $j$ are independent of the spectral-spacing parameter $\gamma$.

Although the $G$-factors can be peaked on curved configurations, the relevant summation range for the bulk spin $j_{\text {blk }}$ scales with $\sqrt{j}$. The oscillatory phase factor can, therefore, average out the expectation value for the deficit angle, see Fig. 1. To avoid this, we need to make sure the oscillations are sufficiently slow

$$
\sigma\left(\frac{S_{\mathrm{ARC}}}{\ell_{P}^{2}}\right)=\frac{1}{\ell_{P}^{2}} \frac{\partial\left(S_{\mathrm{ARC}}\right)}{\partial j_{\mathrm{blk}}} \times \sigma\left(j_{\mathrm{blk}}\right) \sim \gamma \epsilon \sqrt{j} \stackrel{!}{\lesssim} \mathcal{O}(1) .
$$

Thus, whereas the scaling for the deficit angle (12) requires a choice of larger $j,(13)$ demands that with growing $j$ we choose smaller $\gamma$, and thus a smaller spacing between the area eigenvalues. Taking $j$ large and keeping $\gamma$ fixed - as for the large $j$-limit discussed above and often treated in the literature - the phase factor will oscillate more and more strongly and suppress the configurations on which the $G$-factors are peaked, see Fig. 1. These expectations are confirmed by numerical examples in the supplemental material [41].

We note that $\gamma$ enters (13) simply because $S_{\text {ARC }}$ is linear in the $a$ and $\partial a / \partial j \sim \gamma$ from (1). Depending on which quantity we consider fixed, we can also interpret (13) as a bound on the curvature per triangle $\epsilon \lesssim 1 /(\gamma \sqrt{j})$, or a $\gamma$ - and curvature dependent upper bound on the spin $j$.

We have considered the simplest triangulation that differentiates between LRC and ARC. As we only employ scaling arguments, similar conclusions may also apply for larger triangulations. In future work we will investigate

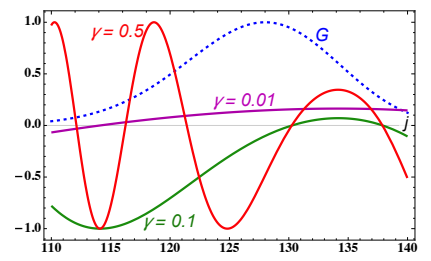

(a) $j=99.5$

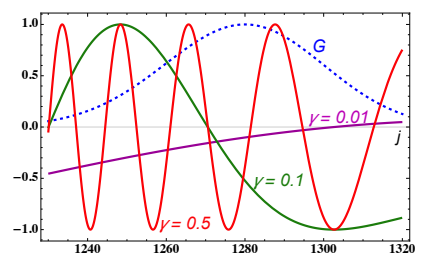

(b) $j=999.5$
FIG. 1: The $G$-factors (dashed), which impose the matching conditions weakly, and the real part of the product of the amplitude factors $\mathcal{A}_{t}$ and $\mathcal{A}_{\sigma}$ as functions of the bulk spin $j_{\text {blk }}$ for various $\gamma$ (solid lines). Here, the $G$ factors peak on a curvature value $\epsilon \approx 0.5$. Larger $\gamma$ 's lead to a more oscillatory behaviour. These examples are detailed in the supplemental material [41].

examples including bulk edges and vertices. Finally, to reach definite conclusions on the continuum limit it will be necessary to see how the implementation of the constraints changes under coarse graining. The models proposed here simplify this task immensely.

Discussion. Area operators are central in a number of approaches to 4D quantum gravity, notably LQG and holography. To achieve a quantum dynamics that reproduces GR, constraints between the areas need to hold. This is, however, hindered if areas have an asymptotically equispaced spectrum and are locally independent.

The imposition of these constraints is pivotal in spin foam quantization. This leads to involved amplitudes, which has so far prevented satisfactory resolution of key dynamical questions, most pressingly whether the models suppress curvature excitations. Thus we propose to use, instead of the standard spin foam amplitudes, a class of effective models, with a transparent encoding of the dynamics and much more amenable for numerical investigations $[56,57]$. If it becomes clear that these effective models lead to a gravitational dynamics in the large scale limit, one can study the effects of using more involved versions, including a sum over orientations or degenerate geometries [58]. See [59] for first insights in the 3D case.

In the models proposed here the constraints are imposed weakly, but as strongly as allowed by the LQG Hilbert space, from which the discrete, locally independent area spectra result. Whether this leads to the correct dynamics is not understood, even in much simpler models than gravity, and should be further tested. In particular, for spin foam models, a too weak imposition of the constraints could lead to suppression of curvature.

We have found that curvature is not necessarily suppressed. This result comes with restrictions connecting the average area $a \sim \ell_{P}^{2} \gamma j$, the Barbero-Immirzi parameter $\gamma$, and the curvature $\epsilon_{t}$ per triangle. The concentration of the constraints on a given curvature value improves with growing spin $j$, as $1 / \sqrt{j}$, but is independent 
of $\gamma$. Our condition $\gamma \sqrt{j} \epsilon_{t} \lesssim \mathcal{O}(1)$, prefers small $\gamma$, and hence a small spacing in the area spectrum.

In numerical examples [41], we need large spins and small $\gamma$ to obtain an expectation value for the deficit angle that approximates well the classical value. This justifies our focus on 'effective' models, where we replace the full spin foam simplex amplitude with its large spin asymptotics, which is already obtained in practice around $j=10$, and is given by the cosine (replaced here with the exponential) of the Regge action [11, 55, 60, 61].

It has been argued in [62], that a double scaling limit that takes $\gamma$ small and spins $j$ large, with $\gamma j$ fixed, reproduces the LRC equations of motion. Here, we find also that $\gamma$ should be small and $j$ large, but that we need for the combination $\gamma \sqrt{j} \epsilon_{t}$ to be of order one or smaller. This combination, and the related bound on curvature has also been identified in [63], based on a generalized stationary phase analysis of the EPRL-FK amplitudes. Using much simpler inputs, we have shown that this bound does not depend on specific choices for the spin foam amplitudes. Rather, the reason for this bound is tied to the LQG Hilbert space and the area spectrum it leads to.

The conclusions for the expectation value of the curvature hold in general, but assume that we can control the scale of bulk spin and deficit angles, e.g., via the choice of boundary data. This is not necessarily the case for larger triangulations. To understand the continuum limit, we will have to investigate how these arguments are impacted by coarse graining and renormalization [64]. The investigation of corresponding continuum actions [65], in which the geometricity constraints are also imposed weakly, might elucidate how these constraints behave under renormalization.

The effective model presented here is the numerically fastest spin foam in the literature to date. All the computations for this paper were performed on individual laptops. The recent work [57], which uses the same triangulation, but works with $4 \mathrm{D}$ BF-theory was computed on 32-core machines. No comparable computation has been carried out for the full EPRL-FK models [12]. Effective spin foams should make the study of coarse graining flow [64] more feasible than for other spin foam models and will help to establish whether LQG and spin foams allow for a satisfactory continuum limit.

Acknowledgments. BD thanks Wojciech Kaminski, and BD and HMH thank Abhay Ashtekar, Eugenio Bianchi, Pietro Donà, Aldo Riello, and Simone Speziale for discussions. SKA is supported by an NSERC grant awarded to $\mathrm{BD}$. HMH gratefully acknowledges support from the visiting fellows program at the Perimeter Institute and the warm hospitality of the quantum gravity group. Research at Perimeter Institute is supported in part by the Government of Canada through the Department of Innovation, Science and Economic Development Canada and by the Province of Ontario through the Ministry of Colleges and Universities.

\section{- SUPPLEMENTAL MATERIAL - EFFECTIVE SPIN FOAM MODELS FOR FOUR-DIMENSIONAL QUANTUM GRAVITY}

This supplement consists of a proof of Eq. (6) from the main text (simpler than the one that is currently present in the literature), numerical investigation of the length configurations as the number of discrete area parameters is increased, and a numerical investigation of the simplest triangulation containing a bulk triangle. Equations in the main text are referred to with standard numerals, while those in the supplemental material are preceded by an $S$.

Derivation of the brackets between dihedral angles. The Poisson bracket on $N$ copies of the dual to the Lie algebra of $\mathfrak{s u}(2)$ is

$$
\{f, g\}=\sum_{I=1}^{N} \overrightarrow{J_{I}} \cdot\left(\frac{\partial f}{\partial \vec{J}_{I}} \times \frac{\partial g}{\partial \vec{J}_{I}}\right),
$$

where each of the $\left\{\vec{J}_{I}\right\}_{I=1}^{N}$ can be thought of as an angular momentum vector $\vec{J}_{I}=\left(J_{I 1}, J_{I 2}, J_{I 3}\right)$. Consider the case $N=4$, which gives the phase space of a tetrahedron described in terms of the Minkowski area vectors $\vec{A}_{I}=\gamma \vec{J}_{I}$ whose magnitudes $A_{I}$ are equal to the tetrahedron's face areas and whose directions are normal to the faces. (References [8] provide additional details about this phase space and how it connects to the geometry of the tetrahedron.) The (internal) dihedral angle $\phi_{I K}$ between the triangles labelled by $I$ and $K$ is determined by

$$
\vec{A}_{I} \cdot \vec{A}_{K}=-A_{I} A_{K} \cos \phi_{I K} .
$$

Define $B_{12,23} \equiv\left\{\vec{A}_{1} \cdot \vec{A}_{2}, \vec{A}_{2} \cdot \vec{A}_{3}\right\}$ and note that

$$
B_{12,23}=A_{1} A_{2}^{2} A_{3} \sin \phi_{12} \sin \phi_{12}\left\{\phi_{12}, \phi_{23}\right\},
$$

where we used the derivation property of the bracket. On the other hand, we can make use of Eq. (S1) to compute

$$
\begin{aligned}
B_{12,23} & =\sum_{I} \epsilon_{i j k} J_{I i} \frac{\partial}{\partial J_{I j}}\left(A_{1 m} A_{2 m}\right) \frac{\partial}{\partial J_{I k}}\left(A_{2 n} A_{3 n}\right) \\
& =-\gamma \vec{A}_{1} \cdot\left(\vec{A}_{2} \times \vec{A}_{3}\right)=-\gamma \frac{9}{2} V^{2}
\end{aligned}
$$

where we drew on the following expression for the volume of a tetrahedron $V^{2}=\frac{2}{9} \vec{A}_{1} \cdot\left(\overrightarrow{A_{2}} \times \overrightarrow{A_{3}}\right)$. This volume can be also computed through $V=\frac{2}{3} l_{I K}^{-1} A_{I} A_{K} \sin \phi_{I K}$, where $l_{I K}$ is the length of the edge shared by triangles $I$ and $K$. Using this formula twice gives

$$
B_{12,23}=-\frac{2 \gamma}{l_{12} l_{23}} A_{1} A_{2}^{2} A_{3} \sin \phi_{12} \sin \phi_{23} .
$$

Setting the two expressions (S2) and (S4) equal gives

$$
\left\{\phi_{12}, \phi_{23}\right\}=-\frac{2 \gamma}{l_{12} l_{23}} \text {. }
$$


Finally, noting that $A_{2}=\frac{1}{2} l_{12} l_{23} \sin \alpha_{12,23}$, where $\alpha_{12,23}$ is the angle between the edges with lengths $l_{12}$ and $l_{23}$, yields the result quoted in the text, Eq. (6),

$$
\left\{\phi_{12}, \phi_{23}\right\}=-\gamma \frac{\sin \alpha_{12,23}}{A_{2}} .
$$

Of course, the minus sign in this result is just due to the choice of an increasing index ordering for the bracket.

Counting of length configurations. We consider a triangulation with certain edge lengths chosen to be equal and then compute the number of allowed edge length solutions given locally independent discrete asymptotically equidistant area spectra. In order to see how the number of allowed configurations scales if there are no area constraints we consider just one 4 -simplex with vertices (12345) and $p \in\{2,3,4\}$ length parameters. For $p=2$, we set $l_{i j}=x$ and $l_{i 5}=y$, with $i, j \in\{1,2,3,4\}$. For the $p=3$ case we choose: $l_{i j}=x, l_{m n}=y$ and $l_{i m}=z$ with $i, j \in\{1,2,3\}$ and $m, n \in\{4,5\}$. For $p=4$ we have $l_{i j}=w, l_{i 4}=x, l_{i 5}=y$ and $l_{45}=z$ with $i, j \in\{1,2,3\}$. We count all edge length solutions where the triangle areas take discrete values $A_{t} \in\left\{\frac{1}{2}, 1, \cdots, N\right\}$ for $N \in \mathbb{N}$. The left panel of Figure A1 shows a semilog plot of the number of length solutions for a simplex having $p \in\{2,3,4\}$ length parameters. The number of length configurations scales as $N^{1.03 p} \approx N^{p}$.

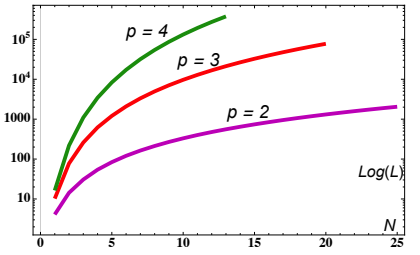

(a)

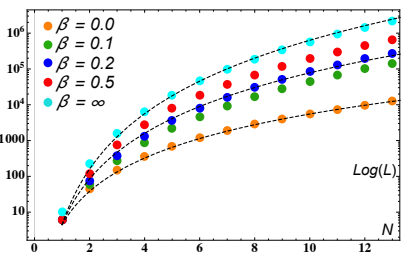

(b)
FIG. A1: Log-linear plots of the number $L$ of length configurations as a function of the maximal area. (a) Count of length configurations with areas up to $N$ in a simplex with $p$ length parameters. (b) The count for two glued simplices with four length parameters. The dashed lines show $N^{3}, N^{4}$ and $N^{5}$ power law scaling.

We also consider a gluing of two simplices with vertices $\sigma=(12345)$ and $\sigma^{\prime}=(12346)$. For the shared tetrahedron we allow two length parameters $u$ for the edges (12) and (34) and $v$ for the remaining four edges. All four areas of the tetrahedron therefore agree, and we are left with one area parameter $a=A(u, v, v)$. Here $A\left(x_{1}, x_{2}, x_{3}\right)$ denotes the area of a triangle with edge lengths $\left(x_{1}, x_{2}, x_{3}\right)$. For the simplex $\sigma$ we introduce additional edge lengths $w$ for edges $(i 5)$ with $i \in\{1,2,3,4\}$. This introduces two more area parameters $b=A(u, w, w)$ and $c=A(v, w, w)$, giving us three length and three area parameters for $\sigma$. We make the same kind of choices for $\sigma^{\prime}$, that is, $w^{\prime}$ gives the length of the edges (i6) leading to area parameters $b^{\prime}=A\left(u, w^{\prime}, w^{\prime}\right)$ and $c^{\prime}=A\left(v, w^{\prime}, w^{\prime}\right)$. After gluing the complex has four length parameters $\left(u, v, w, w^{\prime}\right)$ and five area parameters $\left(a, b, c, b^{\prime}, c^{\prime}\right)$.

We proceed to count the number of configurations with all areas valued in $\left\{\frac{1}{2}, 1, \cdots, N\right\}$, and with maximum deviations given by

$$
\sigma(\Phi)=\beta \sqrt{\ell_{P}^{2} \gamma \frac{\sin \alpha_{v}^{t, \tau}}{a_{t}}}=\beta \sqrt{\frac{\sin \alpha_{v}^{t, \tau}}{\left(j_{t}+\frac{1}{2}\right)}}
$$

for the pairs $\left(\Phi_{e_{i}}^{\tau, \sigma}, \Phi_{e_{i}}^{\tau, \sigma^{\prime}}\right)$ of 3d dihedral angles in the shared tetrahedron $\tau$. This is informed by the deviation Eq. (11) for the $G$-factors. Explicitly we implement the deviation constraints using a Heaviside approximation to the $G$-factors

$$
\left|\Phi_{e_{i}}^{\tau, \sigma}-\Phi_{e_{i}}^{\tau, \sigma^{\prime}}\right|<\sigma(\Phi), \quad i \in\{1,2\} .
$$

We have also introduced a dimensionless parameter $\beta$ to be able to tune between an exact imposition $(\beta=0)$ and no imposition $(\beta=\infty)$ of the matching constraints. The right panel of Fig. A1 shows the results for various choices of $\beta$. For $\beta=0$ we find a scaling $N^{3}$. This is explained by the fact that requiring exact matching forces $w=w^{\prime}$, and thus we have only three parameters. Not imposing the matching conditions, we find a scaling $N^{5}$ reflecting the five area parameters for the two glued simplices. For $\beta \approx 0.15$ we find a scaling of $N^{4}$, see Fig. A1, which is the same as we found for one simplex with $p=4$ free length parameters.

Triangulations with three and with six 4-simplices. Take three 4-simplices with vertices (12345), (12356) and (13456) respectively, and glue these around the shared triangle (135). Here all edges and all but the triangle (135) are in the boundary. Thus we have one bulk triangle and no bulk edges.

We will assume some lengths to be equal, so that we have overall only three length parameters: $x=l_{i j}$, $y=l_{m n}$, and $z=l_{i m}$, with $i, j \in\{1,3,5\}$ and $m, n \in$ $\{2,4,6\}$. Correspondingly, we have three area parameters $a=A(x, x, x), b=A(x, z, z)$, and $c=A(y, z, z)$ where $A\left(x_{1}, x_{2}, x_{3}\right)$ denotes the area of a triangle with lengths $\left(x_{1}, x_{2}, x_{3}\right)$.

Note that with this special choice of boundary data the boundary areas $(b, c)$ do not determine the boundary lengths $(x, y, z)$. To do so one also needs the bulk area a. In Area Angle Regge calculus one has also 3D dihedral angles as boundary data. With the given symmetry reduction, all boundary tetrahedra have the same geometry, determined by edge lengths $(z, y, z, z, x, z)$. We can choose a pair of non-opposite edges, both with length $z$. Due to our choice of symmetric boundary data, the 3D dihedral angles $\phi_{z}$ for the $z$-edges are all the same - thus we have boundary data $\left(b, c, \phi_{z}\right)$. These determine a bulk deficit angle $\epsilon_{a}\left(b, c, \phi_{z}\right)$. 
The matching conditions Eq. (5) for the bulk tetrahedra are all satisfied due to our symmetry reduction. Thus, if we start from the path integral with area and angle variables Eq. (8), and integrate out the bulk 3D dihedral angles, we will just obtain a multiplicative factor, given by the norm of the coherent states $\mathcal{K}_{\tau}(\cdot, \Phi)$.

We can now consider this path integral with a boundary, which, after integrating out the bulk 3D angles, involves only a summation over one spin $j_{a}$.

Alternatively, we can take two such complexes consisting of three 4-simplices each, and glue these so that we obtain a triangulation of $S^{4}$. After integrating out all 3D dihedral angles we will have four area parameters, the areas $b$ and $c$ and the bulk areas $a$ and $a^{\prime}$ from the two complexes respectively. We will compute the expectation value for the deficit angle $\left\langle\epsilon_{a}\right\rangle$-while keeping the areas $\left(a^{\prime}, b, c\right)$ fixed. Classically, i.e., with sharp shapematching constraints and fixed $\left(a^{\prime}, b, c\right)$, these data determine the deficit angles $\epsilon_{a^{\prime}}$ and $\epsilon_{a}$ with $\epsilon_{a}=\epsilon_{a^{\prime}}$.

The summation for the path integral thus involves only the bulk area parameter $a$. There are two contributions to the amplitudes: the exponential of the (Area) Regge action, as well as the inner product $G\left(a, a^{\prime}\right)$ between the coherent states, which impose the matching constraints $\Phi_{z}(a, b, c)=\Phi_{z}\left(a^{\prime}, b, c\right)$. We approximate the factor arising from these inner products between the coherent states by a gaussian

$$
G\left(a, a^{\prime}\right)=\exp \left(-\frac{9}{2 \sigma^{2}(\Phi)}\left(\Phi_{z}(a, b, c)-\Phi_{z}\left(a^{\prime}, b, c\right)\right)^{2}\right)
$$

with a deviation $\sigma$ determined by the Poisson brackets (6):

$$
\sigma^{2}(\Phi)=\frac{1}{2} \frac{\sin \alpha(a, b, c)}{\left(j_{b}+1 / 2\right)}+\frac{1}{2} \frac{\sin \alpha\left(a^{\prime}, b, c\right)}{\left(j_{b}+1 / 2\right)},
$$

where $\sin \alpha(a, b, c)=2 \sqrt{3} a b /\left(a^{2}+3 b^{2}\right)$. The factor 9 in the exponential arises because we have 9 boundary tetrahedra and therefore 9 inner products.

For the computation of the expectation value $\left\langle\epsilon_{a}\right\rangle\left(a^{\prime}, b, c\right)$ we use

$$
\left\langle\epsilon_{a}\right\rangle\left(a^{\prime}, b, c\right)=\frac{1}{\mathcal{Z}} \sum_{j_{a}} \epsilon_{a} G\left(a, a^{\prime}\right) \prod_{t} \mathcal{A}_{t} \prod_{\sigma} \mathcal{A}_{\sigma}
$$

with

$$
\mathcal{Z}=\sum_{j_{a}} G\left(a, a^{\prime}\right) \prod_{t} \mathcal{A}_{t} \prod_{\sigma} \mathcal{A}_{\sigma}
$$

and $\mathcal{A}_{t}$ and $\mathcal{A}_{\sigma}$ defined below Eq. (7) of the main text.

We use homogeneous boundary areas, that is, choose $b=c$. In this case the angles $\Phi_{z}$ and $\epsilon_{a}$ are given by

$$
\Phi_{z}=\arccos \left(\frac{a^{2}}{3 b^{2}}\right), \quad \text { and }
$$

$$
\epsilon_{a}=2 \pi-3 \arccos \left(\frac{9 b^{2}-7 a^{2}}{9 b^{2}-a^{2}}\right)
$$

note that triangle inequalities imply $a^{2} \leq 3 b^{2}$. The area action for the complex with three simplices is

$$
\begin{aligned}
S=9 b\left[\frac{a}{9 b} \epsilon_{a}+2 \pi\right. & -\arccos \left(\frac{3 b^{2}-2 a^{2}}{6 b^{2}-2 a^{2}}\right) \\
& \left.-\arccos \left(\frac{a^{2}}{\sqrt{27 b^{4}-12 a^{2} b^{2}+a^{4}}}\right)\right],
\end{aligned}
$$

and the $G$-function is given in (S7) and (S8) with $b=c$.

The resulting expectation values are shown in Tables I and II. Here we have set $j_{b}=j_{c}=j$. Thus the pair $\left(j, j_{a^{\prime}}\right)$ determine the scale as well as the deficit angle $\epsilon_{a^{\prime}}$. Classically we have $\epsilon_{a}=\epsilon_{a^{\prime}}$. To reproduce this result for the expectation value we need a sufficiently large scale $j$ and a sufficiently small value for the Barbero-Immirzi parameter $\gamma$, in particular if we consider data leading to a small deficit angle.

\begin{tabular}{|c|c|c|c|}
\hline$\left(j+\frac{1}{2}, j_{a^{\prime}}+\frac{1}{2}, \epsilon_{a^{\prime}}\right)$ & $\gamma=0.01$ & $\gamma=0.1$ & $\gamma=0.5$ \\
\hline$(30,38.5,0.52)$ & $0.78-0.03 \mathrm{i}$ & $0.68-0.26 \mathrm{i}$ & $0.17-0.32 \mathrm{i}$ \\
$(100,128,0.54)$ & $0.62-0.062 \mathrm{i}$ & $0.55-0.19 \mathrm{i}$ & $0.17-0.27 \mathrm{i}$ \\
$(300,384,0.54)$ & $0.57-0.02 \mathrm{i}$ & $0.51-0.17 \mathrm{i}$ & $0.16-0.25 \mathrm{i}$ \\
$(1000,1280,0.54)$ & $0.55-0.01 \mathrm{i}$ & $0.50-0.16 \mathrm{i}$ & $0.16-0.24 \mathrm{i}$ \\
\hline
\end{tabular}

TABLE I: Expectation value for the deficit angle $\epsilon_{a}$ with classical value $\approx 0.5$ for various $j, j_{a}^{\prime}$, and $\gamma$.

\begin{tabular}{|c|c|c|c|}
\hline$\left(j+\frac{1}{2}, j_{a^{\prime}}+\frac{1}{2}, \epsilon_{a^{\prime}}\right)$ & $\gamma=0.01$ & $\gamma=0.1$ & $\gamma=0.5$ \\
\hline$(30,40,0.08)$ & $0.39-0.02 \mathrm{i}$ & $0.33-0.15 \mathrm{i}$ & $0.03-0.14 \mathrm{i}$ \\
$(100,133.5,0.06)$ & $0.14-0.01 \mathrm{i}$ & $0.13-0.05 \mathrm{i}$ & $0.03-0.06 \mathrm{i}$ \\
$(300,400,0.08)$ & $0.11-0.00 \mathrm{i}$ & $0.09-0.03 \mathrm{i}$ & $0.03-0.5 \mathrm{i}$ \\
$(1000,1335,0.06)$ & $0.07-0.00 \mathrm{i}$ & $0.06-0.02 \mathrm{i}$ & $0.02-0.03 \mathrm{i}$ \\
\hline
\end{tabular}

TABLE II: Expectation value for the deficit angle $\epsilon_{a}$ with classical value $\approx 0.07$ for various $j, j_{a}^{\prime}$, and $\gamma$.

In this example the averaging of the deficit angle with the $G\left(a, a^{\prime}\right)$ factor (but without the $\mathcal{A}_{t}$ and $\mathcal{A}_{\sigma}$ factors) tends to over-estimate the curvature angle. This is due to a certain asymmetry in the example that partially originates with the generalized triangle inequalities, which restrict $a$ to $a \leq \frac{3}{2} b=\frac{3}{2} c$. The oscillatory behavior of the $\mathcal{A}_{t}$ and $\mathcal{A}_{\sigma}$ factors tends to average out the expectation values - more so for larger Barbero-Immirzi parameter $\gamma$, which leads to more oscillations over the interval where $G\left(a, a^{\prime}\right)$ is sufficiently large, see Fig. A2 and Fig. 1 (in the main text). Note that the expectation values do have imaginary contributions. These arise as the $G\left(a, a^{\prime}\right)$ factor peaks away from the stationary point of the action (where $\epsilon_{a}=0$ ), so the imaginary parts do not average 
out. As the imaginary contributions are sourced by the oscillatory behaviour of the amplitudes, they grow with $\gamma$. Having imaginary contributions on the order of the real contributions indicates that the regime is unreliable, even if the (real part of the) expectation value happens to be near the classical value.

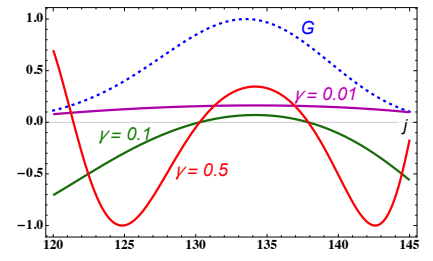

(a) $j=99.5$

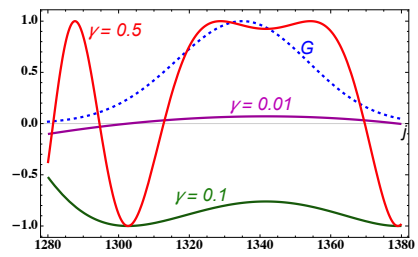

(b) $j=999.5$
FIG. A2: The $G\left(a, a^{\prime}\right)$ factor (dashed) and the real part of the product of the amplitude factors $\mathcal{A}_{t}$ and $\mathcal{A}_{\sigma}$ as a function of $j_{a}$ for $\epsilon_{a^{\prime}} \sim 0.07$ and different $\gamma$-values.

[1] G. Ponzano and T. Regge, "Semiclassical limit of Racah coefficients," in: Spectroscopic and group theoretical methods in physics, p1-58, ed. F. Bloch, North-Holland Publ. Co., Amsterdam, (1968).

[2] H. Waelbroeck and J. A. Zapata, "Translation symmetry in 2+1 Regge calculus," Class. Quant. Grav. 10 (1993) 1923.

[3] V. Bonzom and B. Dittrich, "Dirac's discrete hypersurface deformation algebras," Class. Quant. Grav. 30 (2013) 205013 [arXiv:1304.5983 [gr-qc]].

[4] B. Dittrich and P. A. Höhn, "From covariant to canonical formulations of discrete gravity," Class. Quant. Grav. 27 (2010) 155001 [arXiv:0912.1817 [gr-qc]]; B. Dittrich and P. A. Höhn, "Canonical simplicial gravity," Class. Quant. Grav. 29 (2012) 115009 [arXiv:1108.1974 [gr-qc]].

[5] A. Ashtekar, "New Variables for Classical and Quantum Gravity," Phys. Rev. Lett. 57 (1986) 2244.

[6] C. Rovelli and L. Smolin, "Discreteness of area and volume in quantum gravity," Nucl. Phys. B 442 (1995) 593 Erratum: [Nucl. Phys. B 456 (1995) 753] [grqc/9411005].

[7] A. Ashtekar and J. Lewandowski, "Quantum theory of geometry. 1: Area operators," Class. Quant. Grav. 14 (1997) A55 [gr-qc/9602046]; A. Ashtekar and J. Lewandowski, "Quantum theory of geometry. 2. Volume operators," Adv. Theor. Math. Phys. 1 (1998) 388 [gr-qc/9711031].

[8] E. Bianchi and H. M. Haggard, "Discreteness of the volume of space from Bohr-Sommerfeld quantization," Phys. Rev. Lett. 107 (2011) 011301, [arXiv:1102.5439 [gr-qc]]; E. Bianchi and H. M. Haggard, "Bohr-Sommerfeld Quantization of Space," Phys. Rev. D 86 (2012) 124010, [arXiv:1208.2228 [gr-qc]].

[9] W. Wieland, "Fock representation of gravitational boundary modes and the discreteness of the area spectrum," Annales Henri Poincare 18 (2017) no.11, 3695 [arXiv:1706.00479 [gr-qc]].
[10] A. Perez, "The Spin Foam Approach to Quantum Gravity," Living Rev. Rel. 16 (2013) 3 [arXiv:1205.2019].

[11] J. W. Barrett and L. Crane, "Relativistic spin networks and quantum gravity," J. Math. Phys. 39 (1998) 3296 [gr-qc/9709028].

[12] J. Engle, R. Pereira and C. Rovelli, "The Loop-quantumgravity vertex-amplitude," Phys. Rev. Lett. 99 (2007) 161301 [arXiv:0705.2388 [gr-qc]]; L. Freidel and K. Krasnov, "A New Spin Foam Model for 4d Gravity," Class. Quant. Grav. 25 (2008) 125018 [arXiv:0708.1595 [grqc]]; E. R. Livine and S. Speziale, "Consistently Solving the Simplicity Constraints for Spinfoam Quantum Gravity," EPL 81 (2008) no.5, 50004 [arXiv:0708.1915 [gr-qc]]; J. Engle, E. Livine, R. Pereira and C. Rovelli, "LQG vertex with finite Immirzi parameter," Nucl. Phys. B 799 (2008) 136 [arXiv:0711.0146 [gr-qc]]; M. Dupuis and E. R. Livine, "Holomorphic Simplicity Constraints for 4d Spinfoam Models," Class. Quant. Grav. 28 (2011) 215022 [arXiv:1104.3683 [gr-qc]]; A. Baratin and D. Oriti, "Group field theory and simplicial gravity path integrals: A model for Holst-Plebanski gravity," Phys. Rev. D 85 (2012) 044003 [arXiv:1111.5842 [hep-th]].

[13] G. 't Hooft, "Dimensional reduction in quantum gravity," Conf. Proc. C 930308 (1993) 284 [gr-qc/9310026].

[14] L. Smolin, "Four principles for quantum gravity," Fundam. Theor. Phys. 187 (2017) 427 [arXiv:1610.01968].

[15] S. Ryu and T. Takayanagi, "Holographic Derivation of Entanglement Entropy from the anti-de Sitter Space/Conformal Field Theory Correspondence," Phys. Rev. Lett. 96 (2006) 181602.

[16] E. Bianchi and R. C. Myers, "On the architecture of spacetime geometry," Class. Quant. Grav. 31 (2014) 214002 [arXiv:1212.5183 [hep-th]].

[17] J. D. Bekenstein and V. F. Mukhanov, "Spectroscopy of the quantum black hole," Phys. Lett. B 360 (1995) 7 [gr-qc/9505012].

[18] A. Ashtekar, J. Baez, A. Corichi and K. Krasnov, "Quantum geometry and black hole entropy," Phys. Rev. Lett. 80 (1998) 904 [gr-qc/9710007].

[19] J. D. Bekenstein, "Statistics of black hole radiance and the horizon area spectrum," Phys. Rev. D 91 (2015) no.12, 124052 [arXiv:1505.03253 [gr-qc]].

[20] J. F. Barbero G. and A. Perez, "Quantum Geometry and Black Holes," arXiv:1501.02963 [gr-qc].

[21] F. Conrady and L. Freidel, "On the semiclassical limit of 4d spin foam models," Phys. Rev. D 78 (2008) 104023 [arXiv:0809.2280 [gr-qc]].

[22] V. Bonzom, "Spin foam models for quantum gravity from lattice path integrals," Phys. Rev. D 80 (2009) 064028 [arXiv:0905.1501 [gr-qc]].

[23] F. Hellmann and W. Kaminski, "Holonomy spin foam models: Asymptotic geometry of the partition function," JHEP 1310 (2013) 165 [arXiv:1307.1679 [gr-qc]].

[24] M. Han, "On Spinfoam Models in Large Spin Regime," Class. Quant. Grav. 31 (2014) 015004 [arXiv:1304.5627].

[25] J. R. Oliveira, "EPRL/FK Asymptotics and the Flatness Problem," Class. Quant. Grav. 35 (2018) no.9, 095003 [arXiv:1704.04817 [gr-qc]].

[26] P. Donà, F. Gozzini and G. Sarno, "Searching for classical geometries in spin foam amplitudes: a numerical method," arXiv:1909.07832 [gr-qc].

[27] E. Bianchi, J. Engle, S. Speziale, ILQGS seminar (March 3rd 2020): Panel on the status of the vertex, Slides

[28] A. Barbieri, "Quantum tetrahedra and simplicial 
spin networks," Nucl. Phys. B 518 (1998) 714 [grqc/9707010]. J. C. Baez and J. W. Barrett, "The Quantum tetrahedron in three-dimensions and fourdimensions," Adv. Theor. Math. Phys. 3 (1999) 815 [grqc/9903060].

[29] E. Bianchi, P. Donà, and S. Speziale, "Polyhedra in loop quantum gravity," Phys. Rev. D 83 (2011) 044035 [arXiv:1009.3402 [gr-qc]].

[30] T. Regge, "General Relativity Without Coordinates," Nuovo Cim. 19 (1961) 558.

[31] J. W. Barrett, M. Rocek and R. M. Williams, "A Note on area variables in Regge calculus," Class. Quant. Grav. 16 (1999) 1373 [gr-qc/9710056].

[32] R. M. Williams, "Recent progress in Regge calculus," Nucl. Phys. Proc. Suppl. 57 (1997) 73 [gr-qc/9702006]; J. Makela, "Variation of area variables in Regge calculus," Class. Quant. Grav. 17 (2000) 4991 [gr-qc/9801022]; J. Makela and R. M. Williams, "Constraints on area variables in Regge calculus," Class. Quant. Grav. 18 (2001) L43 [gr-qc/0011006].

[33] J. W. Barrett, "First order Regge calculus," Class. Quant. Grav. 11 (1994) 2723 [hep-th/9404124].

[34] B. Bahr and B. Dittrich, "Regge calculus from a new angle," New J. Phys. 12 (2010) 033010 [arXiv:0907.4325].

[35] B. Dittrich and S. Speziale, "Area-angle variables for general relativity," New J. Phys. 10 (2008) 083006 [arXiv:0802.0864 [gr-qc]].

[36] S. K. Asante, B. Dittrich and H. M. Haggard, "The Degrees of Freedom of Area Regge Calculus: Dynamics, Non-metricity, and Broken Diffeomorphisms," Class. Quant. Grav. 35 (2018) no.13, 135009 [arXiv:1802.09551]

[37] L. C. Brewin and A. P. Gentle, "On the convergence of Regge calculus to general relativity," Class. Quant. Grav. 18 (2001) 517 [gr-qc/0006017].

[38] These functions depend on a discrete parameter that accounts for multiple roots appearing in this inversion. This parameter is another summation variable for the (constrained) ARC path integral. To simplify notation we suppress it here.

[39] B. Dittrich and J. P. Ryan, "Phase space descriptions for simplicial 4d geometries," Class. Quant. Grav. 28 (2011) 065006 [arXiv:0807.2806 [gr-qc]]; B. Dittrich and J. P. Ryan, "Simplicity in simplicial phase space," Phys. Rev. D 82 (2010) 064026 [arXiv:1006.4295 [gr-qc]]; B. Dittrich and J. P. Ryan, "On the role of the BarberoImmirzi parameter in discrete quantum gravity," Class. Quant. Grav. 30 (2013) 095015 [arXiv:1209.4892 [gr-qc]].

[40] This Poisson bracket (with $\gamma=1$ ) appears in the Kapovich-Millson phase space [42], which is used to describe the space of shapes of tetrahedra with fixed areas $[8,29,43,44]$. The non-commutativity of the angles is inherited by the lengths $[39,45]$.

[41] See Supplemental Material at link for more detailed description.

[42] M. Kapovich and J. J. Millson, "The symplectic geometry of polygons in Euclidean space," J. Diff. Geom. 44 (1996) 479.

[43] F. Conrady and L. Freidel, "Quantum geometry from phase space reduction," J. Math. Phys. 50 (2009) 123510 [arXiv:0902.0351 [gr-qc]].

[44] L. Freidel and S. Speziale, "Twisted geometries: A geometric parametrisation of $\mathrm{SU}(2)$ phase space," Phys. Rev. D 82 (2010) 084040 [arXiv:1001.2748 [gr-qc]].

[45] E. Bianchi, "The length operator in Loop Quantum
Gravity," Nuc. Phys. B 807 (2009) 591.

[46] B. Bahr, B. Dittrich and S. Steinhaus, "Perfect discretization of reparametrization invariant path integrals," Phys. Rev. D 83 (2011) 105026 [arXiv:1101.4775 [gr-qc]]; B. Dittrich and S. Steinhaus, "Path integral measure and triangulation independence in discrete gravity," Phys. Rev. D 85 (2012) 044032 [arXiv:1110.6866 [gr-qc]]; B. Dittrich, W. Kaminski and S. Steinhaus, "Discretization independence implies non-locality in 4D discrete quantum gravity," Class. Quant. Grav. 31 (2014) no.24, 245009 [arXiv:1404.5288 [gr-qc]]; B. Bahr and S. Steinhaus, "Numerical evidence for a phase transition in $4 \mathrm{~d}$ spin foam quantum gravity," Phys. Rev. Lett. 117 (2016) no. 14,141302

[47] F. Girelli, H. Pfeiffer and E. M. Popescu, "Topological Higher Gauge Theory - from BF to BFCG theory," J. Math. Phys. 49 (2008) 032503 [arXiv:0708.3051 [hep-th]].

[48] A. Mikovic and M. Vojinovic, "Poincare 2-group and quantum gravity," Class. Quant. Grav. 29 (2012) 165003 [arXiv:1110.4694 [gr-qc]]; M. Vojinovic, "Causal Dynamical Triangulations in the Spincube Model of Quantum Gravity," Phys. Rev. D 94 (2016) no.2, 024058 [arXiv:1506.06839 [gr-qc]].

[49] S. K. Asante, B. Dittrich, F. Girelli, A. Riello and P. Tsimiklis, "Quantum geometry from higher gauge theory," arXiv:1908.05970 [gr-qc].

[50] A. Baratin and L. Freidel, "Hidden Quantum Gravity in 4-D Feynman diagrams: Emergence of spin foams," Class. Quant. Grav. 24 (2007) 2027 [hepth/0611042]. A. Baratin and L. Freidel, "A 2-categorical state sum model," J. Math. Phys. 56 (2015) no.1, 011705 [arXiv:1409.3526 [math.QA]].

[51] E. R. Livine and S. Speziale, "A New spinfoam vertex for quantum gravity," Phys. Rev. D 76 (2007) 084028 [arXiv:0705.0674 [gr-qc]].

[52] V. Bonzom and E. R. Livine, "Generating Functions for Coherent Intertwiners," Class. Quant. Grav. 30 (2013) 055018 [arXiv:1205.5677 [gr-qc]].

[53] L. Freidel and J. Hnybida, "A Discrete and Coherent Basis of Intertwiners," Class. Quant. Grav. 31 (2014) 015019 [arXiv:1305.3326 [math-ph]].

[54] These are given by a coherent state $\mathcal{K}_{\tau}$ for each boundary tetrahedron.

[55] J. W. Barrett and R. M. Williams, "The Asymptotics of an amplitude for the four simplex," Adv. Theor. Math. Phys. 3 (1999) 209 doi:10.4310/ATMP.1999.v3.n2.a1 [grqc/9809032]; J. W. Barrett and C. M. Steele, "Asymptotics of relativistic spin networks," Class. Quant. Grav. 20 (2003) 1341 [gr-qc/0209023]; J. W. Barrett, R. J. Dowdall, W. J. Fairbairn, H. Gomes and F. Hellmann, "Asymptotic analysis of the EPRL four-simplex amplitude," J. Math. Phys. 50 (2009) 112504 doi:10.1063/1.3244218 [arXiv:0902.1170 [gr-qc]]; J. W. Barrett, R. J. Dowdall, W. J. Fairbairn, F. Hellmann and R. Pereira, "Lorentzian spin foam amplitudes: Graphical calculus and asymptotics," Class. Quant. Grav. 27 (2010) 165009 doi:10.1088/02649381/27/16/165009 [arXiv:0907.2440 [gr-qc]]; M. X. Han and M. Zhang, "Asymptotics of Spinfoam Amplitude on Simplicial Manifold: Euclidean Theory," Class. Quant. Grav. 29 (2012) 165004 [arXiv:1109.0500 [gr-qc]].

[56] S. Speziale, "Boosting Wigner's $n j$-symbols," J. Math. Phys. 58 (2017) no.3, 032501 [arXiv:1609.01632 [grqc]]; P. Donà and G. Sarno, "Numerical meth- 
ods for EPRL spin foam transition amplitudes and Lorentzian recoupling theory," Gen. Rel. Grav. 50 (2018) 127 [arXiv:1807.03066 [gr-qc]]; P. Donà, M. Fanizza, G. Sarno and S. Speziale, "Numerical study of the Lorentzian Engle-Pereira-Rovelli-Livine spin foam amplitude," Phys. Rev. D 100 (2019) no.10, 106003;

[57] P. Donà, F. Gozzini, G. Sarno, "Numerical analysis of spin foam dynamics and the flatness problem," [arXiv:2004.12911].

[58] J. Engle, "Proposed proper Engle-Pereira-Rovelli-Livine vertex amplitude," Phys. Rev. D 87 (2013) no.8, 084048 [arXiv:1111.2865 [gr-qc]]; J. Engle, "A spin-foam vertex amplitude with the correct semiclassical limit," Phys. Lett. B 724 (2013) 333 [arXiv:1201.2187 [gr-qc]].

[59] B. Dittrich, C. Goeller, E. Livine and A. Riello, "Quasilocal holographic dualities in non-perturbative 3d quantum gravity I - Convergence of multiple approaches and examples of Ponzano-Regge statistical duals," Nucl. Phys. B 938 (2019) 807 [arXiv:1710.04202 [hep-th]]; B. Dittrich, C. Goeller, E. R. Livine and A. Riello, "Quasi-local holographic dualities in non-perturbative 3d quantum gravity II - From coherent quantum boundaries to $\mathrm{BMS}_{3}$ characters," Nucl. Phys. B 938 (2019) 878 [arXiv:1710.04237 [hep-th]]; B. Dittrich, C. Goeller, E. R. Livine and A. Riello, "Quasi-local holographic dualities in non-perturbative 3d quantum gravity," Class. Quant. Grav. 35 (2018) no.13, 13LT01 [arXiv:1803.02759 [hep-th]]; C. Goeller, E. R. Livine and A. Riello, "NonPerturbative 3D Quantum Gravity: Quantum Boundary States and Exact Partition Function," Gen. Rel. Grav. 52 (2020) no.3, 24 [arXiv:1912.01968 [hep-th]].

[60] W. Kaminski, M. Kisielowski and H. Sahlmann, "Asymptotic analysis of the EPRL model with timelike tetrahedra," Class. Quant. Grav. 35 (2018) no.13, 135012 [arXiv:1705.02862 [gr-qc]].

[61] P. Donà, M. Fanizza, G. Sarno, and S. Speziale, "SU(2) graph invariants, Regge actions and polytopes," Class. Quant. Grav. 35 (2018) 045011 [arXiv:1708.01727].
[62] E. Magliaro and C. Perini, "Regge gravity from spinfoams," Int. J. Mod. Phys. D 22 (2013) 1 [arXiv:1105.0216 [gr-qc]]; E. Magliaro and C. Perini, "Emergence of gravity from spinfoams," EPL 95 (2011) no.3, 30007 [arXiv:1108.2258 [gr-qc]].

[63] M. Han, "Semiclassical Analysis of Spinfoam Model with a Small Barbero-Immirzi Parameter," Phys. Rev. D 88 (2013) 044051 [arXiv:1304.5628 [gr-qc]].

[64] B. Bahr and B. Dittrich, "Improved and Perfect Actions in Discrete Gravity," Phys. Rev. D 80 (2009) 124030 [arXiv:0907.4323 [gr-qc]]; B. Dittrich, "The continuum limit of loop quantum gravity - a framework for solving the theory," arXiv:1409.1450 [gr-qc]; B. Dittrich, S. Mizera and S. Steinhaus, "Decorated tensor network renormalization for lattice gauge theories and spin foam models," New J. Phys. 18 (2016) no.5, 053009 [arXiv:1409.2407 [gr-qc]]; B. Dittrich and M. Geiller, "Flux formulation of loop quantum gravity: Classical framework," Class. Quant. Grav. 32 (2015) no.13, 135016 [arXiv:1412.3752 [gr-qc]]; B. Dittrich, E. Schnetter, C. J. Seth and S. Steinhaus, "Coarse graining flow of spin foam intertwiners," Phys. Rev. D 94 (2016) no.12, 124050 [arXiv:1609.02429 [gr-qc]]; C. Delcamp and B. Dittrich, "Towards a phase diagram for spin foams," Class. Quant. Grav. 34 (2017) no.22, 225006 [arXiv:1612.04506 [gr-qc]]; B. Bahr, G. Rabuffo and S. Steinhaus, "Renormalization of symmetry restricted spin foam models with curvature in the asymptotic regime," Phys. Rev. D 98 (2018) no.10, 106026 [arXiv:1804.00023 [gr-qc]].

[65] K. Krasnov, "Gravity as BF theory plus potential," Int. J. Mod. Phys. A 24 (2009) 2776 [arXiv:0907.4064 [grqc]]; K. Krasnov, "Effective metric Lagrangians from an underlying theory with two propagating degrees of freedom," Phys. Rev. D 81 (2010) 084026 [arXiv:0911.4903 [hep-th]]. 